\title{
Anabases
}

ANABASES Traditions et réceptions de l'Antiquité

2 | 2005

Varia

\section{En guise de présentation (suite)}

\section{Pascal Payen}

\section{OpenEdition}

Journals

Édition électronique

URL : http://journals.openedition.org/anabases/1705

DOI : 10.4000/anabases. 1705

ISSN : 2256-9421

\section{Éditeur}

E.R.A.S.M.E.

Édition imprimée

Date de publication : 1 octobre 2005

Pagination : 221-230

ISSN : 1774-4296

\section{Référence électronique}

Pascal Payen, « En guise de présentation (suite) », Anabases [En ligne], 2 | 2005, mis en ligne le 01 juillet 2011, consulté le 20 octobre 2019. URL : http://journals.openedition.org/anabases/1705; DOI : 10.4000/anabases. 1705

Ce document a été généré automatiquement le 20 octobre 2019.

(c) Anabases 


\title{
En guise de présentation (suite)
}

\author{
Pascal Payen
}

La présente chronique constitue le prolongement de celle qui a paru dans le $\mathrm{n}^{\circ} 1 \mathrm{~d}$ ' Anabases (p.277-282). Le sommaire ci-dessous regroupe le plan de la première livraison (\$1-3) et celui de la seconde (\$4-5). Depuis la première parution, nous avons tenu compte, autant que possible, des publications récentes ainsi que des remarques et critiques qui nous ont été faites. Nous en profitons pour redire que cet «Atelier » est conçu comme un espace d'échanges des informations et de confrontation des hypothèses, et nous invitons tous les chercheurs à proposer à leur tour d'autres chroniques, qui fassent le point sur des publications, des initiatives éditoriales, ou qui ouvrent le chantier de la réception des historiens anciens vers de nouveaux champs méthodologiques.

Plan des sections parues dans Anabases 1 (2005) et 2 (2005):

1. Prolégomènes

2. La réception des historiens anciens : un champ d'étude à construire

3. La redécouverte de Thucydide comme historien politique

4. Éditions, traductions, commentaires et paratextes : les outils de l'interprétation

5. Connaissance et fonctions des historiens anciens à travers les Histoires anciennes

6. Document et monument : les historiens anciens entre histoire scientifique et histoire des représentations

\section{4. Éditions, traductions, commentaires et paratextes : les outils de l'interprétation}

1 Les travaux des historiens du livre ont montré que la nature du support et l'ensemble des éléments qui accompagnent le texte - avant-propos, préface, dédicace, titres et intertitres, notes, commentaires, lexiques ${ }^{1}$ - affectent le sens et sont inséparables de l'histoire des interprétations d'une œuvre. Dans le cas des historiens, les classements qui ont présidé à l'organisation des grands corpus de fragments ont également orienté, puis pérennisé des interprétations, dont plusieurs recherches récentes commencent à retracer l'histoire ${ }^{2}$. Seules des études de cas, nombreuses et appuyées sur les travaux des historiens de la lecture, sur les acquis de la narratologie et sur la théorie de la 
réception, permettront de comprendre comment furent lus les historiens anciens, quelle place ils occupent dans l'histoire de la réception de l'Antiquité, et pourquoi, à ce titre, ils constituent un jalon nécessaire dans l'histoire de nos rapports au passé. On esquissera ici trois sujets d'étude possibles.

2 4.1. Tout d'abord, que révèlent les éditions et traductions à propos de la connaissance et de l'intérêt qui s'attachent aux classiques de l'historiographie ancienne? Pour l'histoire de la lecture, toute étude devra repartir des travaux de Peter Burke ${ }^{3}$, de Dominique Morineau ${ }^{4}$ et de Chantal Grell ${ }^{5}$. De 1450 à 1700, Salluste est, en Europe, l'historien le plus édité et le plus traduit (282 éditions du Catilina, 271 du Jugurtha), devant Valère-Maxime, César, Quinte-Curce, la Germanie de Tacite, Tite-Live, Suétone. Le premier historien grec, Flavius Josèphe, n'arrive qu'en dixième position et précède Hérodote, Thucydide, Polybe, Xénophon et Diodore, du quinzième au dix-neuvième rang. Au XviII ${ }^{e}$ siècle, dans la hiérarchie d'ensemble des auteurs anciens, les historiens sont nettement devancés par Cicéron, Virgile, Ovide, Horace, chez les auteurs latins, et par Plutarque et Homère. Entre 1670 et 1730, en France, aucun historien grec n'est publié dans sa langue, et les traductions, déjà anciennes, deviennent difficiles à trouver. Il n'existe pas une seule traduction d'Hérodote entre celle de Du Ryer (1645) et le travail de Pierre-Henri Larcher (1786), pas une non plus de Thucydide entre la "belle infidèle " de Nicolas Perrot d'Ablancourt, suivie de celle des Helléniques de Xénophon (1662), et l'édition, avec traduction et commentaire, de Pierre-Charles Lévêque (1795). Xénophon est considéré comme historien, mais avec la Cyropédie, vie romancée de Cyrus l'Ancien, d'inspiration socratique. Les traductions importantes - il faut ajouter celle de Lucien par l'abbé Massieu, entre 1781 et 1787, celle des Vies de Plutarque par l'abbé Ricard, en 1798 - datent de la seconde moitié du xviII ${ }^{e}$ siècle et sont une des marques de la redécouverte des Grecs dans un mouvement plus vaste de retour à l'Antique. Mais elles sont aussi le signe de la méconnaissance croissante des langues anciennes, notamment $\mathrm{du}$ grec, selon une courbe continue depuis l'extrême fin du xvI siècle ${ }^{6}$, par différence avec l'Allemagne, qui privilégie cette langue, les Pays-Bas, autre refuge du grec, notamment au XVIII siècle, et l'Angleterre. Ces aléas trouvent-ils leur source dans une évolution interne à la tradition classique ? En quel sens - négatif pour la France, semble-t-il - a pu jouer la dimension européenne du mouvement des Lumières? L'érudition protestante, active dans l'Europe septentrionale et anglo-saxonne, a-t-elle, concernant les historiens, exercé une influence en France? La tradition scolaire et les réformes souhaitées par Rollin en faveur d'un enseignement de l'histoire, dans son Traité des études (1726-1728), ont-elles suscité un renouveau dans l'édition? On sait, par exemple, que son élève et ami Jean-Baptiste Crévier (1693-1765), qui l'aida à composer son Histoire ancienne (1731-1738) et acheva l'Histoire romaine (1739-1747), donna aussi une édition complète de Tite-Live (1735-1742). Enfin, quelle place occupent les historiens anciens dans le "partage des savoirs ", entre approche historique et discours ethnologique ${ }^{7}$ ? Leurs Américains à eux - ceux que décrit Lafitau, dès 1724, dans Les Mœurs des sauvages américains comparées aux mœurs des premiers temps - sont les Barbares de l'Est, sans parler de ces Barbares de l'intérieur que peuvent être tour à tour les peuples d'Italie, les Messéniens, les Arcadiens, les Romains parfois.

3 4.2. Une seconde incidence majeure sur l'interprétation de l'historiographie classique provient des caractéristiques des grands corpus qui réunissent les centaines de prosateurs dont les œuvres ne sont plus connues que par des fragments d'étendue très variable. Dans le cas des Grecs, l'édition des Fragmenta Historicorum Graecorum, due à 
Karl et Theodor Müller et parue chez Firmin Didot, de 1841 à 1851, a été remplacée, nous l'avons rappelé, par le travail monumental de Felix Jacoby, interrompu en 1958, à la mort du savant, et récemment repris pour être achevé ${ }^{8}$. Or l'édition et le commentaire de Jacoby ne sont pas seulement de nature philologique. Au cours de longues recherches préparatoires, menées dans le premier quart $d u x^{e}$ siècle ${ }^{9}$, le savant allemand a divisé la tradition historiographique grecque en sept grands genres, dont le plus important est constitué par l'« Histoire contemporaine» (Zeitgeschichte), elle-même subdivisée en dix sections à la fois thématiques et chronologiques. Or ce genre aurait atteint presque d'emblée la perfection avec Thucydide, après qu'Hérodote eut accompli, dans son œuvre, le trajet en forme de maturation et de progrès qui conduit de la géographie et de l'ethnographie (livres I-IV), à l'histoire des temps récents, avec la révolte de l'Ionie et les guerres Médiques (livres V-IX). Une telle conception est marquée par l'Altertumswissenschaft du XIX ${ }^{e}$ siècle, qui érige Thucydide en modèle d'une histoire scientifique dont les épigones sont les historiens et antiquisants allemands. Les Fragmente der griechischen Historiker imposent et pérennisent ainsi des catégories, des classements qui appartiennent certes, pour les uns, aux Anciens, mais qui relèvent aussi, pour beaucoup d'autres, de l'histoire de la tradition historiographique postérieure. Celle-ci commence avec les différents canons élaborés à l'époque romaine et tardive, bien étudiés par Roberto Nicolai ${ }^{10}$. Dans une voie similaire, l'histoire de nos propres catégories d'analyse doit être poursuivie audelà de l'Antiquité, en prenant pour objet d'étude les éditions et instruments de travail que nous élaborons, comme l'ont entrepris, à propos des FGrHist, plusieurs contributions d'un colloque consacré à la question des corpus de fragments ${ }^{11}$. Il devrait en résulter une perception plus fine, inédite du moins, de la superposition des théories contemporaines sur l'histoire et des réflexions des historiens anciens.

4 4.3. Les traductions constituent un troisième élément d'importance, encore trop peu étudié, dans l'histoire de la réception des historiens. Dès 1566, Henri Estienne note, dans l'avis «Au lecteur » qui ouvre son Apologie pour Hérodote, que les « historiens tant grecs que latins [sont] ceux entre tous qui sont plus maniés de toutes sortes de gens par le moyen des traductions ». Le cas des traductions françaises d'Hérodote ${ }^{12}$ permet de retracer le développement des deux grandes lignes interprétatives auxquelles on ne cesse de le rattacher, depuis les appréciations divergentes de Thucydide et de Denys d'Halicarnasse : d'un côté, l'historien des conflits politiques et militaires; de l'autre, l'historien des coutumes et des mœurs. Après les traductions latines - partielle pour Guarino, en 1427, puis complète avec Lorenzo Valla, de 1452 à $1456^{13}-$, la première traduction française complète, due à Pierre Saliat, paraît en 1556. L'imprimeur de la réédition en petit format, de 1575, la destine aux « gentilshommes et seigneurs qui font profession des armes ». Estienne, lui, toujours dans l'avis « Au lecteur » et l'adresse « À un sien ami " de L'introduction au Traité de la conformité des merveilles anciennes avec les modernes, privilégie un Hérodote historien des mœurs. Il faut ensuite attendre 1645 et la traduction de Du Ryer, composée à partir de la version latine de Valla. Deux érudits du XVIII ${ }^{\mathrm{e}}$ siècle s'appliquent à un examen critique des traductions d'Hérodote. François Bellenger, dans ses Essais de critique. II. Sur les traductions d'Hérodote (Amsterdam, F. L'Honoré et fils, 1740), et Jean Bouhier, dans des Recherches et dissertations sur Hérodote (Dijon, chez Pierre de Saint, 1746), en multipliant les remarques philologiques et en revenant à des traductions plus littérales donnent à lire un Hérodote enraciné dans les multiples contextes propres aux peuples dont il consigne les coutumes. La traduction et les notes de Larcher (1786) reflètent une sensibilité aux récits de voyages dans le 
Nouveau Monde et un goût pour la comparaison entre les mœurs. Les traductions des $\mathrm{XIX}^{\mathrm{e}}$ et $\mathrm{xx}^{\mathrm{e}}$ siècles intègrent à la fois les progrès de la philologie, mais aussi les préoccupation des États-nations en construction, souvent engagés dans des entreprises coloniales; dans ce contexte, elles élaborent et diffusent, à l'image de celles de Philippe-Ernest Legrand (Paris, Les Belles Lettres, CuF, 1932-1954) et d'Andrée Barguet (Paris, Gallimard, Bibliothèque de la Pléiade, 1964), avant tout un Hérodote historien des guerres Médiques et de leurs prolégomènes. Ces remarques devraient inciter à mener la comparaison avec Thucydide, sans omettre de partir des analyses de leurs premiers lecteurs, en particulier Denys d'Halicarnasse, Cicéron et Lucien, dont la redécouverte influe sur le travail des traducteurs. L'examen conduit sur l'Enquête d'Hérodote suggère deux voies de recherche: d'une part, l'analyse comparée des traductions des historiens, replacées dans les contextes politiques et culturels où elles ont été élaborées; d'autre part, la confrontation entre le discours des traductions et celui du "paratexte» (titre, sous-titres, intertitres, préface, avertissement, notes, bibliographie), inséparable aussi bien de notre perception des classiques que des lois de tous les « genres » historiques.

\section{Connaissance et fonctions des historiens anciens à travers les Histoires anciennes}

5 C'est à partir du deuxième quart du xvIII ${ }^{\mathrm{e}}$ siècle que sont publiées de nombreuses Histoires des Grecs et des Romains. La présentation synthétique de Carmine Ampolo, pour l'histoire grecque ${ }^{14}$, et la thèse de Mouna Raskolnikoff, pour l'histoire romaine ${ }^{15}$, en apportent toutes les preuves. Il reste, notamment pour la Grèce, à mesurer quelle place occupent les historiens, en tant que modèles et autorités, dans cette tradition ininterrompue et en constant renouvellement, en fonction du statut nouveau affecté, pour l'écriture de l'histoire de l'Antiquité, à d'autres corpus documentaires, en particulier l'épigraphie et l'archéologie.

Quelle est, tout d'abord, la courbe des historiens qui apparaissent le plus souvent en tant que modèle rhétorique? Comment les exigences critiques se greffent-elles sur la tradition rhétorique? L'attention devrait porter en premier lieu sur les préfaces et les textes théoriques qui se présentent comme des artes historicae et comme autant de relais entre le Comment il faut écrire l'histoire de Lucien et le dernier grand traité d'histoire humaniste, publié par Mably en 1783 : De la manière d'écrire l'histoire. Le Père Rapin, dans ses Instructions pour l'histoire (1677), privilégie César, Salluste et Thucydide. Le Père Duguet, dans sa Lettre touchant à l'étude des humanités (1684), ajoute aux deux premiers Tite-Live, Quinte-Curce, Tacite et Justin, preuve supplémentaire de l'ignorance quasi générale du grec aux XVII ${ }^{\mathrm{e}}$ et XVIII ${ }^{\mathrm{e}}$ siècles. Fénélon fait exception et recommande, dans sa Lettre à l'Académie (1714), l'étude d'Hérodote, Xénophon, Polybe, Thucydide, Tite-Live, Salluste et Tacite. Tous ces traités recherchent, pour l'écriture historique, la perfection d'un art oratoire, tout en admettant, comme Jean-Baptiste de Rocoles, que "toutes sortes de styles y peuvent être employés ${ }^{16}$ ". De Lucien à Mably, reste ainsi à écrire l'histoire comparée de la place qu'ont occupée les historiens anciens dans les traités théoriques et rhétoriques.

7 L'analyse devrait porter conjointement sur l'utilisation du corpus des Anciens dans la composition des grandes Histoires de l'Antiquité, notamment du côté des Grecs. Ceux-ci, en effet, ont été discrédités par la victoire des Modernes, favorables aux Romains, dans 
l'illustre Querelle. L'Histoire ancienne de Rollin (1731-1738) - consacrée pour les trois quarts aux Grecs - offrirait un bon point d'observation. Rollin pouvait disposer du premier volume de Temple Stanyan, paru en 1707, une histoire de la Grèce, des origines à la fin de la guerre du Péloponnèse ${ }^{17}$, et il n'est pas même assuré qu'il l'ait connue. Rollin fait donc œuvre de pionnier, lorsqu'il affirme que «quiconque aspirera à la réputation de savant sera obligé de voyager, pour ainsi dire, longtemps chez les Grecs ${ }^{18}$ ", et il s'appuie avant tout sur le corpus des historiens qu'il est un des rares à lire dans la langue originale. Les listes et notices qu'il rédige à leur sujet, les notes infrapaginales, avec références et citations du texte grec, semblent montrer que la dimension politique de leurs œuvres tend à passer au premier plan. Ainsi le Thucydide «maître d'éloquence ", que vante Perrot d'Ablancourt dans la préface à sa traduction de 1662, s'efface-t-il au profit d'un Thucydide historien de l'Athènes de Cimon et de Périclès, où le "gouvernement populaire" repose sur les principes de l'«égalité" et de la « liberté ». L'émergence de l'« Athènes bourgeoise ${ }^{19}$ " ne pourrait-elle trouver une de ses sources essentielles, jusqu'ici négligée, dans le travail de Rollin, où s'élabore le nouveau statut d'autorité des historiens grecs? L'étude de l'immense fortune de son Histoire ancienne, traduite en plusieurs langues et utilisée jusque dans les années 1850-1870, serait de nature à conforter cette hypothèse.

De même, la comparaison pourrait s'instaurer à partir de l'importance réservée aux historiens latins parmi les quarante auteurs qui figurent dans les volumes de la collection Ad usum Delphini, parus entre 1674 et 1691, à l'initiative du duc de Montausier, gouverneur du fils de Louis XIV. Treize historiens y figurent, et plus d'une œuvre est éditée dans une intention historique, à l'image de la Correspondance de Cicéron, pourvue d'un index historicus et d'un index à fonction chronologique. Pourtant, en dépit de l'affirmation de Bossuet qui, dans le programme d'éducation destiné au Dauphin, définit l'histoire comme « la maîtresse de la vie humaine et de la politique », ce sont les divergences qui l'emportent, dues au projet lui-même et à la fonction référentielle des historiens dans leur rapport au présent ${ }^{20}$.

\section{Document et monument : les historiens anciens entre histoire scientifique et histoire des représentations}

9 Quel fut l'apport des historiens anciens à la constitution de l'histoire scientifique, au cours du xIX siècle? Ils participent globalement d'une prise de distance par rapport à l'Antiquité, avec laquelle le rapport d'identification, suscité dans le cadre de l'historia magistra vitae, s'efface au fur et à mesure que s'étendent et se précisent les frontières d'un vaste champ d'étude, "la science de l'Antiquité ${ }^{21}$ ». Le regard doit, une fois encore, se tourner vers la Prusse et son université, pour saisir les contextes qui interviennent dans un mouvement qui s'étend ensuite à toute l'Europe, jusqu'à ce que l'écriture et la méthode historiques se transforment de nouveau, face aux sollicitations de la sociologie, de l'anthropologie et des courbes de prix, dans le premier tiers du $\mathrm{xx}^{\mathrm{e}}$ siècle.

10 Le premier élément de contexte est celui, déjà signalé, de la nouvelle fondation de l'Université prussienne, en réaction à l'occupation napoléonienne, après Iéna. La reconstruction est l'œuvre de grands fonctionnaires instruits, produits de l'État et de l' 
Aufklärung implantés en Prusse. En l'absence d'une tradition urbaine où aurait pu se développer une bourgeoisie éclairée, ils formaient une sorte d'avant-garde savante, seule capable de s'opposer à l'aristocratie traditionnelle des Junker ${ }^{22}$. Les créateurs de la nouvelle université sont animés par l'esprit du néo-humanisme qui repose sur un universalisme de la raison, opposé à la fois à l'ancien corporatisme nobiliaire et au nouvel utilitarisme napoléonien.

11 Le modèle de la connaissance historique qui, pour l'Antiquité, se développe dans ce contexte est celui de l'Historismus (ou historicisme), où se trouve mise en avant la nécessité d'une analyse critique des sources primaires transmises avant tout par les États. Ceux-ci sont considérés comme des individualités et comme les "personnages » que doit privilégier toute analyse. Un historien ancien est dès lors moins un littérateur plus ou moins génial qu'un homme écrivant pour sa patrie, ce qui explique le prestige singulier dont jouissent Tite-Live et, plus encore, Thucydide. L'interprétation des historiens de la Grèce et de Rome en termes politiques et patriotiques ne tient pas seulement à l'historicisme; il ressortit aussi au contexte très présent du développement des États-nations et, à leur sujet, du problème majeur de l'unité. À ces combats et à ces réflexions, participent, en Allemagne et ailleurs, la plupart des historiens antiquisants.

12 Une troisième conséquence résulte des deux précédentes, lorsque l'étude de l'Antiquité se constitue, selon le vœu de Wolf, « en un ensemble organique ", qui doit « réunir les différentes disciplines » relatives aux mondes anciens ${ }^{23}$. Ainsi l'Antiquité grecque et romaine pourra-t-elle participer d'une «science philosophico-historique bien ordonnée » et d'une "encyclopédie philologique », dont les termes empruntent aux courants de pensée du $\mathrm{xVIII}^{\mathrm{e}}$ siècle, mais qui portera finalement le nom d' Altertumswissenschaft. La frontière entre " philosophes » et érudits tend à s'estomper et le rapprochement entre eux s'opère au mieux dans la science philologique.

Ces éléments de contexte mériteraient d'être précisés, en fonction de ce qu'il advient des historiens anciens, dans ces débats qui touchent à la construction des sciences historiques, sur une période dont le terme s'étend bien au-delà des infortunes que connaît l'Europe parcourue par les armées de Napoléon. On se risquera à signaler quelques hypothèses.

$14 \mathrm{Au}$ cours du xIX $\mathrm{e}^{\mathrm{e}}$ siècle, les historiens anciens quittent pour l'essentiel le domaine exclusif des Belles Lettres (excepté, peut-être, dans une certaine tradition française). De «monuments", ils deviennent des « documents" parmi d'autres, qui fournissent des renseignements de première valeur sur les États et la politique. Comment un tel changement dans le rapport aux œuvres se traduit-il dans l'expression du point de vue de l'historien moderne, dans l'appareil critique, dans les citations et les reprises nonlittérales de l'œuvre ancienne à l'intérieur du récit ou de l'exposé historique? Quels nouveaux canons se dessinent? Sous quelles formes persistent les anciennes classifications?

L'évolution du statut des historiens anciens suscite des travaux spécifiques de deux ordres, dont l'histoire reste à étudier. Les premières histoires de l'historiographie grecque se détachent difficilement des modèles anciens ${ }^{24}$. L'Essai sur l'historiographie des Romains (1849) de Léon de Closset est placé sous le patronage de Cicéron et du patriotisme ${ }^{25}$. Mais dans le même temps apparaissent de grandes éditions commentées : pour Thucydide les onze volumes de Poppo (1821-1840), repris ensuite par Stahl (1875-1889), et l'édition de Classen et Steup ( $3^{\mathrm{e}}$ à $5^{\mathrm{e}}$ éd. 1900-1922); pour 
Hérodote celle de Stein (1856-1861, augmentée dans l'édition de 1869-1871, elle-même rééditée en 1884). Chez Firmin Didot, paraissent dans le même temps les Fragmenta Historicorum Graecorum (1841-1851).

Ces instruments facilitent l'accès aux textes, désormais imprimés sans exception avec les découpages en livres, chapitres et courtes sections, fixés par les imprimeurs des $\mathrm{XVI}^{\mathrm{e}}$ et XVII ${ }^{\mathrm{e}}$ siècles, et pourvus d'une annotation qui permet de dégager aussitôt les données positives et d'opérer les rapprochements avec des passages parallèles ou avec d'autres sources. De la sorte, l'historien devient un réservoir de "sources» et de "faits », que l'on extrait de son œuvre et dont les références sont indiquées en note. Le contexte narratif et discursif est de plus en plus oublié; les questions d'énonciation notamment dans le cas des discours reconstitués - ne sont guère prises en compte, si bien que sont attribués à l'historien des propos placés dans la bouche d'un personnage ou des opinions rapportées. Ces éléments créent de nombreux effets de distorsion, qu'une histoire de l'interprétation et de la réception propre à chaque historien devrait analyser. De plus, dans le contexte plus large de la science historique, les Anciens sont jugés à l'aune des critères qui appartiennent à un cadre épistémologique différent: primat de la recherche des causes, utilisation de documents écrits, critique des sources, séparation entre histoire, géographie et ethnographie.

L'historien ancien se trouve dès lors appréhendé par ses « collègues » modernes selon les règles de la méthode positiviste, tandis que, du côté des études littéraires, ce sont ses caractéristiques stylistiques et rhétoriques qui sont mises en valeur, dans la tradition issue de Cicéron et de Denys d'Halicarnasse. Dans l'une et l'autre approche, il n'est donc pas lu en fonction des contextes qui lui sont propres. C'est pourtant seulement à ce prix qu'il serait possible de retrouver et le projet de connaissance qui explique la genèse de l'œuvre, et les différentes cadres qui en sont indissociables. Ainsi serait-on conduit à ne plus étudier l'historiographie des Anciens sans prendre en compte les outils éditoriaux et les catégories d'analyse qui nous permettent de les lire, mais bien plutôt à l'intérieur d'une histoire des représentations -, par conséquent à les appréhender à la fois comme document et comme monument.

\section{NOTES}

1. Cf. G. GenETTE, Seuils, Paris, Seuil, 1987.

2. Cf. les actes du colloque de Heidelberg (1995), édités par G. W. MOsT, Collecting Fragments Fragmente sammeln, Göttingen, Vandenhoeck \& Ruprecht, 1997, notamment la section «C. Historical Fragments ", détaillée infra, note 11. D. LENFANT aborde de nombreux problèmes dans la riche introduction de l'édition de Ctésias, qu'elle a publiée dans la CUF, Paris, Les Belles Lettres, 2004, ainsi que dans son article "Peut-on se fier aux 'fragments' d'historiens? L'exemple des citations d'Hérodote", Ktèma 24 (1999), p. 103-121.

3. Cf. P. BURKE, "A survey of the popularity of ancient historians, 1450-1700", History and Theory 5 (1966), p. 135-152. 
4. Cf. D. MORINEAU, La réception des historiens anciens dans l'historiographie française (fin XVII ${ }^{e}$ début XVIII ${ }^{e}$, thèse de doctorat, Paris-IV Sorbonne, s. d. [1988], en particulier p. 342-358, sur les modes d'évaluation des historiens anciens.

5. Cf. Ch. GRELL, Le XVIII siècle et l'Antiquité en France, 1680-1789, Oxford, Voltaire Foundation, $\mathrm{n}^{\circ}$ 330-331, 1995, p. 297-301 (les historiens dans la hiérarchie des auteurs anciens), 307-324 (les traductions), 984-1000 (les historiens au sortir de la Querelle), et passim.

6. La vitalité de l'étude du grec dans la première moitié $\mathrm{du} \mathrm{XVI}^{\mathrm{e}}$ siècle et les raisons de son déclin rapide sont l'objet du livre de J.-C. SALAdin, La bataille du grec à la Renaissance, Paris, Les Belles Lettres, 2000. Pour le latin, la situation n'est en fait guère meilleure, si l'on en juge par le souvenir qu'en gardent les élèves des collèges et le niveau atteint au sortir des études : cf., outre Ch. GRELL, dans le premier chapitre de Le XVIII e siècle et l'Antiquité, les analyses de F. WAQUET, Le latin ou l'empire d'un signe, Paris, Albin Michel, 1998, p. 17-39 notamment.

7. Cf. M. D UCHET, Le partage des savoirs. Discours historique, discours ethnologique, Paris, La Découverte, 1985, p. 7-52.

8. Cf. G. SCHEPENS, J. ENGELS, E. ThreYs, J. BollAnsÉE, FGrHist, IV. Biography and Antiquarian Literature (les fasc. 1, 3 et 7 sont parus), Leiden, Brill, 1998-1999. P. BONNECHÈRE a établi trois volumes d' indices, Leiden, Brill, 1999. Est également annoncée la partie V, consacrée à la géographie historique, sous la direction de H.-J. GEHRKE.

9. Cf. Cf. F. JАСову, "Über die Entwicklung der giechischen Historiographie und den Plan einer neuen Sammlung der griechischen Historikerfragmente", Klio 9 (1909), p. 80-123, repris in Abhandlungen zur griechischen Geschichtsschreibung, Leiden, Brill, 1956, p. 281-341. Sur Felix Jacoby, cf. la brève étude de M. CHAMBers, "Felix Jacoby", in W.W. BRIGgS and W.M. CALDER III (ed. by), Classical Scholarship. A Biographical Encyclopedia, New York and London, Garland Publishing, 1990, p. 205-210, en attendant les travaux conduits notamment par G. SCHEPENS et J. BOLLANSÉE.

10. Cf. R. NicolaI, La storiografia nell'educazione antica, Pisa, Giardini, 1992, p. 250-339.

11. Cf. in G. W. Most (ed.), Collecting Fragments - Fragmente sammeln, les contributions d'A. Grafton, "Fragmenta Historicorum Graecorum. Fragments of some lost Enterprises" (p. 124-143), G. SCHEPENS, “Jacoby's FGrHist : Problems, Methods, Prospects”, p. 144-172, avec une riche bibliographie, et S.C. HuMPHREYS, "Fragments, fetishes, and philosophies : towards a history of greek historiography after Thucydides" (p. 207-224). Apporteront également beaucoup les actes à paraître du colloque international organisé par D.Lenfant: Athénée et les fragments d'historiens (Strasbourg, 16-18 juin 2005), Paris, De Boccard, 2006.

12. Nous nous permettons de renvoyer à notre étude "Hérodote et ses traducteurs français (xvi $\mathrm{e}_{-}$ $\mathrm{xx}^{\mathrm{e}}$ siècles) : histoire politique ou histoire des mœurs ?", Euphrosyne. Rivista de filologia clássica 29 (2001), p. 9-28. Toute étude sur la réception d'Hérodote doit (re)partir des analyses d'A. Momigliano, "La place d'Hérodote dans l'histoire de l'historiographie", in Problèmes d'historiographie antique et moderne, Paris, Gallimard, 1983, p. 169-185 (références, p. 169).

13. Celle-ci est publiée seulement en 1474, à Venise. Elle est rééditée en 1566, à Paris, par Henri Estienne, qui poursuit l'œuvre de son père, Robert Estienne, en faveur des historiens anciens. Sur les raisons qui conduisent Henri Estienne à prendre le parti d'Hérodote, contre Thucydide et Plutarque, et les contextes de polémique religieuse et philosophique qui en constituent le fond, cf. B. Boudou, "La réception d'Hérodote au XVI ${ }^{e}$ siècle", in G. LACHENAUd et D. LonGRÉE (dir.), Grecs et Romains aux prises avec l'histoire. Représentations, récits et idéologie, Rennes, P. U. de Rennes, 2003, p. 731-738.

14. Cf. C. AmPoLo, Storie greche. La formazione della moderna storiografia sugli antichi Greci, Torino, Einaudi, 1997.

15. Cf. M. RASKOLNIKOFF, Histoire romaine et critique historique dans l'Europe des Lumières. La naissance de l'hypercritique dans l'historiographie de la Rome antique, Paris, De Boccard, 1992. 
16. Jean-Baptiste de Rocoles, Introduction générale à l'histoire, Paris, 1661, 1672, t. 1, p. 11, qui ajoute : «Salluste et Tacite sont fort concis. Tite-Live est plus étendu. Florus fort libre et fort fleuri. Thucydide beaucoup plus sévère » (cité par D. MORINEAU, La réception des historiens anciens [n. 4], p. 33).

17. T. STANYAN, The Grecian History from the Origin of Greece to the Death of Philip of Macedon, London, 2 vol., 1707 et 1739 ; traduit, après la mort de Rollin, par Diderot: Histoire de la Grèce, Paris, Briasson, 3 vol., 1743.

18. Charles Rollin, Traité des études, Paris, 1726-1728, t. III, p. 207 (cf. encore t. IV, p. 191-192, et Histoire ancienne, livre 5, p. 188 [in Cuvres complètes, éd. Letronne, Paris, Firmin Didot, 1821-1825, vol. 2, p. 188]).

19. Sur cette histoire, cf. N. LORAUX et P. VIDAL-NAQUET, "La formation de l'Athènes bourgeoise", in

P. VIDAL-NAQUET, La démocratie grecque vue d'ailleurs, Paris, Flammarion, 1990, p. 161-209, 362-383.

20. Pour conduire cette étude, le plus précieux des instruments de travail est le volume dirigé par C. VolPILHAC-Auger, La collection Ad Usum Delphini. L'Antiquité au miroir du Grand Siècle, Grenoble, ELLUG, Université Stendahl, 2000. Cf. nos remarque dans "Un échec éditorial au Grand Siècle : la collection Ad usum Delphini. Notes de lectures", Bulletin de l'Association Guillaume Budé 2001/2, p. 239-251.

21. Ce mouvement correspond à l'émergence du régime moderne d'historicité et à l'effacement de l'historia magistra vitae : cf. l'ouvrage, déjà signalé dans la précédente chronique, de F. HARTOG, Régimes d'historicité, Paris, Seuil, 2003, p. 68-107 notamment.

22. Cf. Th. NIPPERDEY, "La Prusse et l'université", in Réflexions sur l'histoire allemande (1982), trad. fr. de C. Orsini, Paris, Gallimard, 1992, p. 198-221.

23. Cf. B. B RAvo, Philologie, histoire, philosophie de l'histoire. Étude sur J.G. Droysen historien de l'Antiquité, Varsovie, 1968, rééd. Hildesheim-Zürich-New York, Georg Olms Verlag, 1988, p. 71-72. 24. Le premier à analyser en termes historiques la tradition historiographique grecque est, à notre connaissance, F. CREUZER, Die historische Kunst der Griechen in ihrer Entstehung und Fortbildung, Leipzig, G.J. Göschen, 1803, 2ème éd. in Deutsche Schriften, III, 1, Leipzig und Darmstadt, C.W. Leske, 1845.

25. Léon DE CLOSSET, Essai sur l'historiographie des Romains jusqu'au siècle d'Auguste, Bruxelles, 1849. La formule cicéronienne Memoria vitae est placée en épigraphe, et l'historiographie est définie comme « l'exposé de la manière dont un peuple conçoit sa propre vie » (p. II).

\section{AUTEUR}

\section{PASCAL PAYEN}

payen@univ-tlse2.fr 